PROCEEDINGS OF THE

AMERICAN MATHEMATICAL SOCIETY

Volume 133, Number 9, Pages 2737-2739

S 0002-9939(05)07824-X

Article electronically published on March 22, 2005

\title{
ALMOST-DISJOINT CODING AND STRONGLY SATURATED IDEALS
}

\author{
PAUL B. LARSON
}

(Communicated by Alan Dow)

\begin{abstract}
We show that Martin's Axiom plus $\mathfrak{c}=\aleph_{2}$ implies that there is no $\left(\aleph_{2}, \aleph_{2}, \aleph_{0}\right)$-saturated $\sigma$-ideal on $\omega_{1}$.
\end{abstract}

Given cardinals $\lambda, \kappa$ and $\gamma$, a $\sigma$-ideal $I$ on a set $X$ is said to be $(\lambda, \kappa, \gamma)$-saturated if for every set $\left\{A_{\alpha}: \alpha<\lambda\right\} \subset \mathcal{P}(X) \backslash I$ there exists a set $Y \in[\lambda]^{\kappa}$ such that for all $Z \in[Y]^{\gamma}, \bigcap\left\{A_{\alpha}: \alpha \in Z\right\} \notin I$. Laver [6] was the first to show the consistency of an $\left(\aleph_{2}, \aleph_{2}, \aleph_{0}\right)$-saturated ideal on $\omega_{1}$, using a huge cardinal. Shelah [10] later showed that the nonstationary ideal on $\omega_{1}$ restricted to a given stationary set can be $\left(\aleph_{2}, \aleph_{2}, \aleph_{0}\right)$-saturated, using a supercompact cardinal.

The cardinal characteristic ap is defined to be the least $\kappa$ such that there exist an almost disjoint family $\left\{e_{\alpha}: \alpha<\kappa\right\}$ (i.e., each $e_{\alpha}$ is an infinite subset of $\omega$, and for each distinct pair $\alpha, \beta<\kappa, e_{\alpha} \cap e_{\beta}$ is finite) and a set $A \subset \kappa$ such that for no $x \subset \omega$ does it hold for all $\alpha<\kappa$ that $\alpha \in A$ if and only if $e_{\alpha} \cap x$ is infinite (in [5] we called this $\mathfrak{q}$, but 1 shows that we should not have, as consistently every set of reals of cardinality $\mathfrak{a p}$ is a $\mathrm{Q}$-set). We let $\mathfrak{c}$ denote the cardinality of the continuum. It follows easily that $2^{\gamma}=\mathfrak{c}$ for every infinite $\gamma<\mathfrak{a p}$.

Given a cardinal $\gamma, \mathrm{MA}_{\gamma}$ is the variant of Martin's Axiom that says that if $P$ is a c.c.c. partial order and $D_{\alpha}(\alpha<\gamma)$ are dense subsets of $P$, then there is a filter $G \subset P$ such that $G \cap D_{\alpha}$ is nonempty for each $\alpha<\gamma$. It is a standard fact that $\mathrm{MA}_{\gamma}$ implies that $\mathfrak{a p}>\gamma$ [4].

In this note, we show that the statement $\mathfrak{a p}=\mathfrak{c}=\aleph_{2}$ implies that there is no countably complete $\left(\aleph_{2}, \aleph_{2}, \aleph_{0}\right)$-saturated $\sigma$-ideal on $\omega_{1}$. This contradicts statements in [7, 8, to the effect that the axiom PFA (see [10]) had been shown to be consistent with the existence of a stationary subset of $\omega_{1}$ such that the nonstationary ideal restricted to this set is $\left(\aleph_{2}, \aleph_{2}, \aleph_{0}\right)$-saturated. This situation is addressed by Nyikos in [9] and in another corrigendum to appear.

For a fixed cardinal $\kappa$, an ideal on a set $X$ is $\kappa$-dense if there is a subset $\mathcal{A}$ of $\mathcal{P}(X) \backslash I$ of cardinality $\kappa$ such that every $I$-positive subset of $X$ contains a member of $\mathcal{A}$ modulo $I$. It follows easily that every $\aleph_{1}$-dense $\sigma$-ideal is $\left(\aleph_{2}, \aleph_{2}, \aleph_{0}\right)$-saturated.

Received by the editors May 9, 2003 and, in revised form, May 14, 2004.

2000 Mathematics Subject Classification. Primary 03E50; Secondary 54D15.

The research in this paper was conducted with the support of a FAPESP fellowship (Grant \# 02/11551-3) at the University of São Paulo.

(C)2005 American Mathematical Society Reverts to public domain 28 years from publication 
It is a classical fact due to Ulam that there is no $\aleph_{0}$-dense $\sigma$-ideal on $\omega_{1}$ (see, for instance, Lemma 10.13 of [3]). Taylor [11] showed that under $\mathrm{MA}_{\aleph_{1}}$ there is no $\aleph_{1}$-dense $\sigma$-ideal on $\omega_{1}$. The proof of Theorem 18 in [2] shows that $\mathfrak{a p}>\aleph_{1}$ suffices, i.e., that the following holds (a version of the argument appears also in [5]).

Fact 0.1. If $\mathfrak{a p}>\aleph_{1}$, then there is no $\aleph_{1}$-dense $\sigma$-ideal on $\omega_{1}$.

For the rest of this note, we fix an almost disjoint family $\left\{e_{\alpha}: \alpha<\omega_{1}\right\}$. For each $n \in \omega$ and each (possibly finite) $\sigma \subset \omega$, we let $E_{\sigma}^{n}=\left\{\alpha<\omega_{1}|| e_{\alpha} \cap \sigma \mid \geq n\right\}$, and we let $F_{\sigma}^{n}=\omega_{1} \backslash E_{\sigma}^{n}$.

Lemma 0.2. Assume that $\mathfrak{a p}>\aleph_{1}$. Let I be a $\sigma$-ideal on $\omega_{1}$ and let $\beta$ be a cardinal such that $\mathcal{P}\left(\omega_{1}\right) / I$ is not $\beta$-dense. Let $\left\{A_{\alpha}: \alpha<\beta\right\}$ be a subset of $\mathcal{P}\left(\omega_{1}\right) \backslash I$. Then there exist an $x \subset \omega$ and an $n \in \omega$ such that

- $F_{x}^{n} \notin I$,

- for each $\alpha<\beta$ there exists an $m \in \omega$ such that $E_{x \cap m}^{n} \cap A_{\alpha} \notin I$.

Proof. Since $\mathcal{P}\left(\omega_{1}\right) / I$ is not $\beta$-dense, we may fix $\left\{B_{\alpha}: \alpha<\beta\right\} \subset \mathcal{P}\left(\omega_{1}\right) \backslash I$ and $D \in \mathcal{P}\left(\omega_{1}\right) \backslash I$ such that each $B_{\alpha} \subset A_{\alpha}$ and each $B_{\alpha} \cap D=\emptyset$. Since ap $>\aleph_{1}$, there exists an $x \subset \omega$ such that $e_{\gamma} \cap x$ is infinite for each $\gamma \in \bigcup\left\{B_{\alpha}: \alpha<\beta\right\}$ and $e_{\gamma} \cap x$ is finite for each $\gamma \in D$. Since $D \subset \bigcup\left\{F_{x}^{n}: n<\omega\right\}$, we may fix an $n \in \omega$ such that $F_{x}^{n} \notin I$. Similarly, for each $\alpha<\beta$, since $B_{\alpha} \subset \bigcup\left\{E_{x \cap m}^{n}: m<\omega\right\}$, there is an $m \in \omega$ such that $E_{x \cap m}^{n} \cap A_{\alpha} \notin I$.

The following theorem shows that $\mathfrak{a p}>\aleph_{1}$ implies that there is no $\sigma$-ideal $I$ on $\omega_{1}$ which is $\left(\gamma, \gamma, \aleph_{0}\right)$-saturated, where $\gamma$ is the least cardinality of a dense subset of $\mathcal{P}\left(\omega_{1}\right) / I$. In particular, if $\mathfrak{a p}=\mathfrak{c}=\aleph_{2}$, then there is no $\left(\aleph_{2}, \aleph_{2}, \aleph_{0}\right)$-saturated $\sigma$-ideal on $\omega_{1}$.

Theorem 0.3. Assume that $\mathfrak{a p}>\aleph_{1}$, and let I be a $\sigma$-ideal on $\omega_{1}$. Let $\gamma$ be the least cardinal such that there exists a dense (modulo I) subset of $\mathcal{P}\left(\omega_{1}\right) \backslash I$ of cardinality $\gamma$. Then there is a sequence $\left\langle D_{\alpha}: \alpha<\gamma\right\rangle$ of members of $\mathcal{P}\left(\omega_{1}\right) \backslash I$ such that for every cofinal $X \subset \gamma$ there exists a countable $y \subset X$ such that $\bigcap\left\{D_{\alpha}: \alpha \in y\right\} \in I$.

Proof. Let $\left\{A_{\alpha}: \alpha<\gamma\right\}$ enumerate a dense subset of $\mathcal{P}\left(\omega_{1}\right) \backslash I$ modulo $I$. For each $\beta<\gamma$, apply Lemma 0.2 to $\left\{A_{\alpha}: \alpha<\beta\right\}$, obtaining $x_{\beta}, n_{\beta}$ and $D_{\beta}=F_{x_{\beta}}^{n_{\beta}}$ such that $D_{\beta} \notin I$ and such that for each $\alpha<\beta$ there exists an $m \in \omega$ such that $A_{\alpha} \cap E_{x_{\beta} \cap m}^{n_{\beta}} \notin I$.

Now let $X \subset \gamma$ be cofinal. Let $Z$ be the set of pairs $(n, \sigma)(n \in \omega, \sigma \subset \omega$ finite) such that there exists a $\beta \in X$ with $E_{\sigma}^{n} \cap D_{\beta} \in I$. We claim that $\left\{E_{\sigma}^{n}:(n, \sigma) \in Z\right\}$ is predense in $\mathcal{P}\left(\omega_{1}\right) \backslash I$, i.e., that for every $\alpha<\gamma$ there exist a $\beta \in X$, an $n \in \omega$ and a finite $\sigma \subset \omega$ such that $E_{\sigma}^{n} \cap D_{\beta} \in I$ and $E_{\sigma}^{n} \cap A_{\alpha} \notin I$. To verify this, fix $\alpha<\gamma$ and let $\beta$ be any member of $X$ greater than $\alpha$. Then $D_{\beta}=F_{x_{\beta}}^{n_{\beta}}$ and there exists an $m$ such that $E_{x_{\beta} \cap m}^{n_{\beta}} \cap A_{\alpha} \notin I$, so $\beta, n_{\beta}$ and $x_{\beta} \cap m$ suffice for $\alpha$.

Now, for each $(n, \sigma) \in Z$, choose $\beta_{(n, \sigma)} \in X$ such that $E_{\sigma}^{n} \cap D_{\beta_{(n, \sigma)}} \in I$. Then since $\left\{E_{\sigma}^{n}:(n, \sigma) \in Z\right\}$ is predense in $\mathcal{P}\left(\omega_{1}\right) \backslash I, \bigcap\left\{D_{\beta_{(n, \sigma)}}:(n, \sigma) \in Z\right\} \in I$.

We do not know whether some forcing axiom implies that the nonstationary ideal on $\omega_{1}$ is not $\left(\aleph_{2}, \aleph_{1}, \aleph_{0}\right)$-saturated. On the other hand, for all we know, some forcing axiom implies that the nonstationary ideal on $\omega_{1}$ is $\left(\aleph_{2}, \aleph_{1}, \aleph_{0}\right)$-saturated. 


\section{REFERENCES}

[1] J. Brendle, Dow's principle and Q-sets, Canad. Math. Bull. 42 (1999), no. 1, 13-24 MR 1695894 (2000h:03093)

[2] M. Foreman, M. Magidor, S. Shelah, Martin's Maximum, saturated ideals, and non-regular ultrafilters. Part I, Annals of Mathematics 127 (1988), 1-47 MR0924672 (89f:03043)

[3] T. Jech, Set Theory, The third millennium edition, revised and expanded. Springer Monographs in Mathematics. Springer-Verlag, Berlin, 2003 MR 1940513 (2004g:03071)

[4] R.B. Jensen, R.M. Solovay, Some applications of almost disjoint sets, Mathematical Logic and Foundations of Set Theory (Proc. Internat. Colloq., J erusalem, 1968), North-Holland, Amsterdam, pp. 84-104, 1970 MR.0289291(44:6482)

[5] P. Larson, A uniqueness theorem for iterations, J. Symbolic Logic 67 (2002), no. 4, 1344-1350 MR:1955241 (2003m:03081)

[6] R. Laver, An $\left(\aleph_{2}, \aleph_{2}, \aleph_{0}\right)$-saturated ideal on $\omega_{1}$, Logic Colloquium '80 (Prague, 1980), pp. 173-180, Stud. Logic Foundations Math., 108, North-Holland, Amsterdam-New York, 1982. MR0673792 (84a:03060)

[7] P. Nyikos, Complete normality and metrization theory of manifolds, Top. Appl. 123 (1) (2002), 181-192 MR.1921659 (2003f:54048)

[8] P. Nyikos, Applications of some strong set-theoretic axioms to locally compact $T_{5}$ and hereditarily scwH spaces, Fund. Math. 176 (2003), no. 1, 25-45 MR1971471|(2004k:54008)

[9] P. Nyikos, Correction to: "Complete normality and metrization theory of manifolds" [Topology Appl. 123 (2002), no. 1, 181-192], Topology Appl. 138 (2004), no. 1-3, 325-327 MR2035491 (2004k:54030)

[10] S. Shelah, Proper and improper forcing, Perspectives in Mathematical Logic, Springer-Verlag, Berlin, 1998 MF 1623206 (98m:03002)

[11] A.D. Taylor, Regularity properties of ideals and ultrafilters, Ann. Math. Logic 16 (1979), no. 1, 33-55 MF0530430 (83b:04003)

Department of Mathematics, Miami University, Oxford, Ohio 45056

E-mail address: larsonpb@muohio.edu 